\author{
Muhammad Rabiul Islam \\ Md. Mahmudul Hasan Akib \\ Fariha Tabassum \\ Khandakar Akhter Hossain
}

http://dx.doi.org/10.21278/brod72304

ISSN 0007-215X

eISSN $1845-5859$

\title{
ON THE INVESTIGATION OF WIND GENERATED WAVES IN BANGLADESH RIVERS FOR THE ASSESSMENT OF STABILITY REQUIREMENTS IN INLAND VESSEL DESIGN
}

\author{
UDC 629.5.015.1:629.55:551.466.3
}

Original scientific paper

\begin{abstract}
Summary
Standard environmental condition is one of the main inputs in designing a vessel especially in assessment of stability condition. The performance based minimum stability requirements are determined by assessing vessels' dynamic failure modes. Winds as well as wind generated waves are the main factors that affect a specific vessel's dynamics. Wind generated waves in rivers though are usually small in comparison with ocean waves may play a crucial role behind inland vessels accidents. The river condition of a crucial location in Bangladesh inland river routes is assessed where wind velocities have been taken for a specific duration from a reliable secondary source. A narrow fetch model that considers the wave generation in off-wind direction for estimating wind wave parameters has been used to consider the spiral shape of Bangladesh inland routes. The Bretschneider energy spectrum model for short term wave state is compared with the fetch limited model JONSWAP for the estimated wave condition. This study indicates the rationality of conforming the safety level of Bangladesh inland vessels equivalent to river-sea vessels as defined by other nationals and the classification societies. The wave parameters that are estimated in this study can be used to form a limited wave scatter table for predicting short term environmental conditions to assess the dynamic stability failure modes of the vessels.
\end{abstract}

Key words: wind wave; inland vessel; stability; fetch; energy spectrum; significant wave height; peak period

\section{Introduction}

The dynamics of a ship is completely governed by the combination of ship dimensions, internal loads and external/environmental loads. The environmental loads are mainly due to wind and waves. The waves can be confined to wind generated waves in case of inland waterways. The IMO 2008 Intact Stability (IS) code [1] considers the environmental loads by its 'Severe wind and rolling criterion (weather criterion)' where steady wind pressure, ship roll due to wave action and gusty wind pressure are the main characteristic parameters. 
Muhammad Rabiul Islam, Md. Mahmudul Hasan Akib, Fariha Tabassum, Khandaker Akhter Hossain
On the Investigation of Wind Generated Waves in Bangladesh Rivers for the Assessment of Stability Requirements in Inland Vessel Design

Among them the ship roll due to wave action is again a function of bilge keel, breadth to draught $(\mathrm{B} / \mathrm{d})$ ratio, block coefficient, ship rolling period and wave steepness. Wave steepness is defined as the ratio of wave height to wavelength. Interim guidelines on the 'Second Generation Intact Stability Criteria' (SGISC) were approved by the Maritime Safety Committee (MSC) of IMO at a session held on from 13 to 22 May, 2020 [2]. Performancebased criteria for assessing five dynamic stability failure modes in waves have been provided in the guidelines, namely, dead ship condition, excessive acceleration, pure loss of stability, parametric rolling and surf-riding/broaching. To decide the performance based minimum stability requirement, the committee had to assume a set of standard environmental conditions. In the guideline, the short-term characterization is given in terms of the Bretschneider wave energy spectrum of sea elevation and the long-term characterization is given in terms of a wave scatter table.

Passenger vessel accidents are recurrent phenomena in Bangladesh inland waterways. Thousands of people have passed away due to these catastrophic incidents. Iqbal et al [3] analyzed the Bangladesh inland passenger vessels accident data of 25 years and found that, in $49 \%$ cases the failure was due to loss of intact stability. The analysis shows that excessive external/environmental loads due to stormy weather conditions were behind $60 \%$ of intact ship accidents. The statistical analysis shows that, mostly the passenger vessels of medium length (around $40 \mathrm{~m}$ ) suffered accidents due to inclement weather/northwester in Bangladesh [4]. So, the wave action of limited wave size on these medium size inland vessels cannot be neglected without a comprehensive investigation which indicates that the dynamic failure modes need to be assessed in case of Bangladesh inland vessels. This kind of assessment is not at all possible without knowing the standard environmental loading. On the other hand, if environmental loading of seaways are considered in the design of inland vessels, very conservative results may be acquired which may not be a reasonable design. The intensity of wave action will depend on the wave dimensions or steepness which again depends on the wind velocity and duration. The appropriate estimation of the wind velocity is of prime importance in ship design. The aim of this study is to estimate the wind generated wave parameters in Bangladesh inland waterways for assessing the minimum stability requirement in designing the domestic ships.

Since no wave data is available for Bangladesh inland navigational routes, wave forecasting procedure on the basis of wind speed and fetch length is considered. The Coastal Engineering Research Center of US Army Corps of Engineers has published a series of research papers $[5,6,7]$ on wind wave estimating methods. A remarkable work in this regard was carried out by Ozeren et al. [8] where a proposal has been made for predicting wind driven waves in small reservoirs. One year wave data of Belgian coast and three year wave data of the coastal area of France was analyzed by Chatelier et al [9] aiming to investigate the stability and sea-keeping performance of river-sea vessels.

A critical location of Bangladesh inland river routes has been chosen where several accidents were recorded in stormy weather. A wave estimation model namely 'NARFET' (a language based computer program) [6] is used in this study as it is quick and inexpensive but considers the complexity of fetch geometry. The energy based significant wave height and peak period have been estimated using the JONSWAP experiment model and the 'NARFET' improved model. In the JONSWAP model simple fetch is considered whereas in the improved 'NARFET' model fetch geometry is taken into account by the inclusion of wave generation in off wind direction. 
On the Investigation of Wind Generated Waves in Bangladesh Rivers for the Assessment of Stability Requirements in Inland Vessel Design
Muhammad Rabiul Islam, Md. Mahmudul Hasan Akib, Fariha Tabassum, Khandaker Akhter Hossain

\section{Bangladesh Inland Vessels Stability Rule and IS Code, 2008}

The current Bangladesh inland vessels stability rule is exactly the same as The International Code on Intact Stability (2008 IS Code). It is noticed that the present stability criteria defined in Bangladesh inland stability rule is established by following the IMO 2008 IS code despite the special features of Bangladesh inland waterways like less water depth, restricted navigational channel, different wind load, sudden seasonal storm etc., which corresponds to a different environmental loading than the seaways. Additionally it is noticed that, no clear guideline is mentioned regarding the calculation of ship roll due to wave action which can be identified as the greatest weakness of Bangladesh Stability rules. In 2008 IS Code, the calculation of this wave action is clearly proposed on the basis of a table of wave steepness data against the rolling period of a ship considering the wind speed $26 \mathrm{~m} / \mathrm{s}$ in seaways. On the basis of work of Sverdrup and Munk [10], Yamagata [11] has proposed this relationship considering the ship's natural roll period is equal to the wave period as the ships suffer resonant roll motion. The relationship between wave steepness and ship natural roll period for different wind speed has been shown in Table 1.

Table 1 Relation between roll period and wave steepness at different wind speeds

\begin{tabular}{|c|c|}
\hline Wind Speed $(\mathbf{m} / \mathbf{s})$ & Relation between wave steepness $(s)$ and roll period $(T)$ \\
\hline 15 & $s=0.155-0.0130 T$ \\
\hline 19 & $s=0.153-0.0100 T$ \\
\hline 26 & $s=0.151-0.0072 T$ \\
\hline
\end{tabular}

Table 2 Wind speeds at different levels of signals in Bangladesh inland navigational route

\begin{tabular}{|c|c|c|}
\hline Signal Name & Wind Speed $(\mathbf{K m} / \mathbf{h})$ & Wind Speed $(\mathbf{m} / \mathbf{s})$ \\
\hline Cautionary Signal No. I & 60 & 16.67 \\
\hline Warning Signal No. II & 61 & 16.94 \\
\hline Danger Signal No. III & 75 & 20.83 \\
\hline Great Danger Signal No. IV & 89 & 24.72 \\
\hline
\end{tabular}

It is written in the Bangladesh stability rules as a precautionary instruction that all kinds of inland passenger vessels must stop their operation if the wind velocity is more than $10 \mathrm{~m} / \mathrm{s}$. This is a quite impracticable instruction which is usually not followed by the passenger vessel operators. Bangladesh inland river authority declares four different levels of signal to the vessel operators on the basis of observed wind speeds. The wind speeds correspond to the different river signals in Bangladesh inland navigational route have been shown in Table 2. The specified wind pressure in the Bangladesh stability rules for deciding the severe wind and rolling criterion (weather criterion) is $0.0322 \mathrm{ton} / \mathrm{m}^{2}$ or $316 \mathrm{~Pa}$. From the following relationship between wind pressure and wind velocity [12], the corresponding wind velocity of the specified wind pressure is $20.5 \mathrm{~m} / \mathrm{s}$ which is a logical one for Bangladesh inland navigational routes and also very close to the wind speed $(20.83 \mathrm{~m} / \mathrm{s})$ that corresponds to danger signal (No. III) of Bangladesh inland navigational routes.

$$
P=\frac{1}{2} \rho C_{D} V_{w}^{2}
$$

In the equation $1, P$ is the wind pressure, $\rho$ the air density $\left(1.225 \mathrm{~kg} / \mathrm{m}^{3}\right), C_{D}$ the $\operatorname{drag}$ coefficient (1.22) and $V_{w}$ is the wind velocity. According to the Bangladesh River Signals (Table 2), the Great Danger Signal IV corresponds to wind velocity of $24.72 \mathrm{~m} / \mathrm{s}$. As per the 
Muhammad Rabiul Islam, Md. Mahmudul Hasan Akib, Fariha Tabassum, Khandaker Akhter Hossain
On the Investigation of Wind Generated Waves in Bangladesh Rivers for the Assessment of Stability Requirements in Inland Vessel Design

recent operational instruction of Bangladesh inland navigation, vessels are to stop operating much before the generation of such dangerous wind. So consideration of the same wave steepness data as proposed in IS Code, 2008 may not be reasonable for Bangladesh inland vessels stability rules. Even if the wind velocity $26 \mathrm{~m} / \mathrm{s}$ is considered in design for a conservative point of view, the wave dimensions will be different in the restricted waterways of Bangladesh.

\section{Water Wave Forecasting}

When the wind is blowing and the waves are growing in response, the water surface tends to be random i.e., the water waves are irregular. Irregular waves are usually treated by spectral methods or by wave train analysis. Spectral approaches are currently the most appropriate method for analyzing a time dependent, three dimensional water surface record. It determines the distribution of wave energy and average of statistical data for each wave frequency by converting the time series of the wave record into a wave spectrum [5]. A five parameter spectrum for fetch limited condition which is known as JONSWAP (Joint North Sea Wave Project) spectrum [13] can reasonably be applied for inland navigational routes. Three of these parameters are usually kept constant. The JONSWAP spectrum can be expressed as:

$$
\begin{aligned}
& E(\omega)=\frac{\alpha g^{2}}{\omega^{5}} \exp \left[-1.25\left(\frac{\omega}{\omega_{p}}\right)^{-4}\right] \gamma^{\exp \left[-\frac{\left(\omega-\omega_{p}\right)^{2}}{2 \sigma^{2} \omega_{p}^{2}}\right]} \\
& \omega_{p}=2 \pi \frac{3.5 F^{-0.33} g^{0.67}}{U_{10}^{0.33}} ; \quad \alpha=0.076\left[\frac{g F}{U_{10}^{2}}\right]^{-0.22} ; \\
& \sigma=0.07 \text { for } \omega \leq \omega_{p} \text { and } \sigma=0.09 \text { for } \omega>\omega_{p}
\end{aligned}
$$

In the above equations, $\alpha$ is the scaling parameter, $\gamma$ the peak enhancement factor (may vary from 1 to 7$), \omega_{p}$ the circular frequency at the spectral peak, $U_{10}$ the wind speed at the elevation $10 \mathrm{~m}$ above the sea surface and $F$ is the fetch length. The JONSWAP spectrum is an extension of the one parameter Pierson - Moskowitz (PM) spectrum which is applicable for fully developed water wave and can be expressed as follows:

$$
E(f)=\frac{0.0081 g^{2}}{(2 \pi)^{4} f^{5}} \exp \left(-0.74\left[\frac{2 \pi U_{w} f}{g}\right]^{-4}\right)
$$

In equation (3) $U_{w}$ is the wind speed at $19.5 \mathrm{~m}$ above mean sea level. In the Interim guidelines on the Second Generation Intact Stability Criteria, the short term characterization of standard environmental conditions has been assumed by Bretschneider energy spectrum. This is a two-parameter spectrum that permits period and wave height to be assigned separately and has the form:

$$
E(\omega)=0.1687 H_{S}^{2} \frac{\omega_{S}^{4}}{\omega^{5}} \exp \left[-0.675\left(\frac{\omega_{S}}{\omega}\right)^{4}\right]
$$

In equation (4), $\omega_{S}=2 \pi / T_{s}$ and $T_{s}=0.946 T_{p}$, where $T_{s}$ and $T_{p}$ are significant wave period and peak period respectively, $H_{s}$ is the significant wave height. The relation between 
On the Investigation of Wind Generated Waves in Bangladesh Rivers for the Assessment of Stability Requirements in Inland Vessel Design
Muhammad Rabiul Islam, Md. Mahmudul Hasan Akib, Fariha Tabassum, Khandaker Akhter Hossain

the peak period $\left(T_{p}\right)$ and the average zero crossing wave period $\left(T_{Z}\right)$ can be obtained as $T_{Z}=$ $0.784 T_{p}$.

\section{Fetch Limited and Fetch Restricted Water Waves}

The parameters of wind waves (height, length, and period) are governed by the fetch, the wind speed, the duration, the distance the wave travelled after generation and the water depth. Wind waves are of three types namely fully developed, fetch limited and duration limited where the latter two types are generated if the wind is unable to impart its maximum energy to the waves. Wind may fail to impart its maximum energy if it cannot blow over a sufficient distance (fetch limited) or cannot get sufficient time (duration limited). The fetch length is the distance travelled by wind across open water and fetch is defined as a region in which the wind speed and direction are reasonably constant. Wind-wave generation in inland navigational routes is generally limited by the geometry of the river, which is very irregular in Bangladesh. Bangladesh is a country which covers approximately $60 \%$ of the largest river delta of the world. In open ocean fetch widths are of the same order of magnitude as the fetch lengths and only the length, not the shape of the fetch, is enough to consider in study of fetch limited waves. But in case of geometrically limited river bodies like in Bangladesh, fetch widths are restricted. Fetch lengths can be measured from the shoreline to the point of interest following the direction of wind. The Shore Protection Manual (SPM, 1984) recommends applying this straight-line fetch length (simple fetch) in forecasting the wave parameters giving no consideration to the fetch geometry. Other methods that are proposed for restricted fetch wave generation including the model 'NARFET' which has been used in this study take into account the fetch geometry by considering fetch lengths in off-wind directions. Within the defined fetch, the wind direction variations should be less than 15 degree and wind speed variations should be less than $2.5 \mathrm{~m} / \mathrm{sec}$ (5.0 knots) from the mean.

\section{Wind Data and Location of Interest}

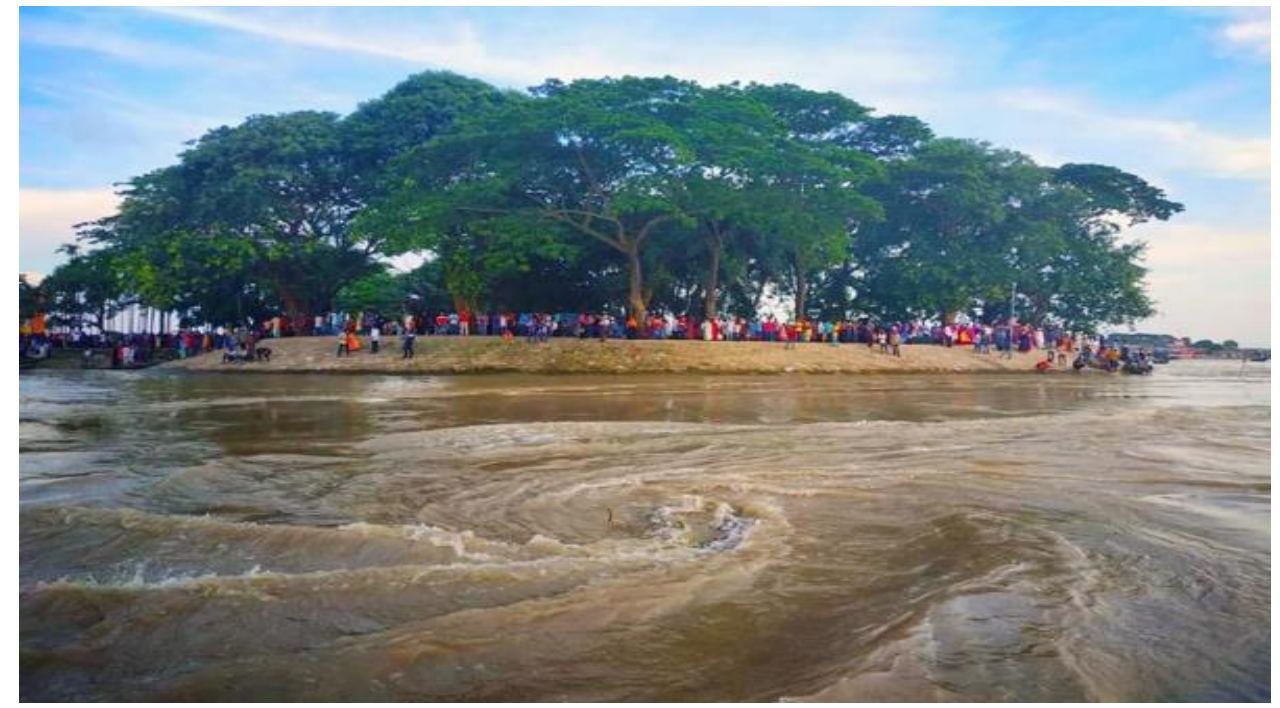

Fig. 1 Formed whirlpool in Chandpur triangle

Bangladesh Meteorological Department (BMD) is responsible for weather forecasting and recording in Bangladesh. But the archived data of this department are difficult to use as these are three-hourly data. On the other hand physical measurement of wind speed could not be managed. So for the wind speed data, a secondary source namely www.windy.com (premium/paid version) [14] has been used and it was possible to pick 01 hour interval data of 
Muhammad Rabiul Islam, Md. Mahmudul Hasan Akib, Fariha Tabassum, Khandaker Akhter Hossain
On the Investigation of Wind Generated Waves in Bangladesh Rivers for the Assessment of Stability Requirements in Inland Vessel Design

forecasted wind speed. Among the four different forecasting models that are offered by windy, the ECMWF (European Center for Medium-Range Weather Forecasts) model data has been used in this study as windy itself says about ECMWF, 'very accurate model'. One of the reasons behind this accuracy may be the comparatively denser grid points which are located at $9 \mathrm{~km}$ distances. The ECMWF data of 27 years were analyzed by Vinayak et al [15] and the reliability of this model data was proven for the wind speed along with other wave parameters. As the site of wave generation, the most critical location in Bangladesh inland waterways has been chosen, which is known as Chandpur Triangle (Figure 1). The three biggest rivers Padma, Meghna and Dakatia have confluence here and occasionally formed a whirlpool of strong current. This location is also known as Bermuda Triangle of Bangladesh as it is a deathtrap for the domestic vessels and there are records of many horrible vessel accidents in this place.

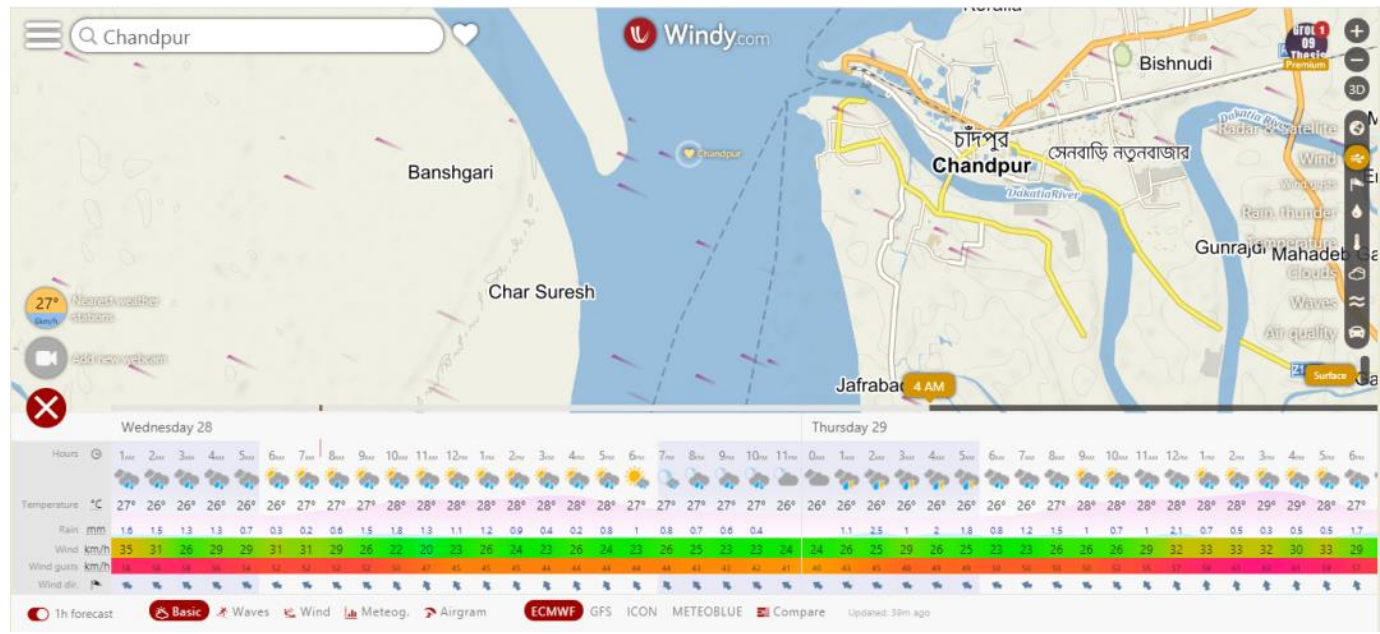

Fig. 2 Forecasted wind velocity at 10 m elevation (www.windy.com)

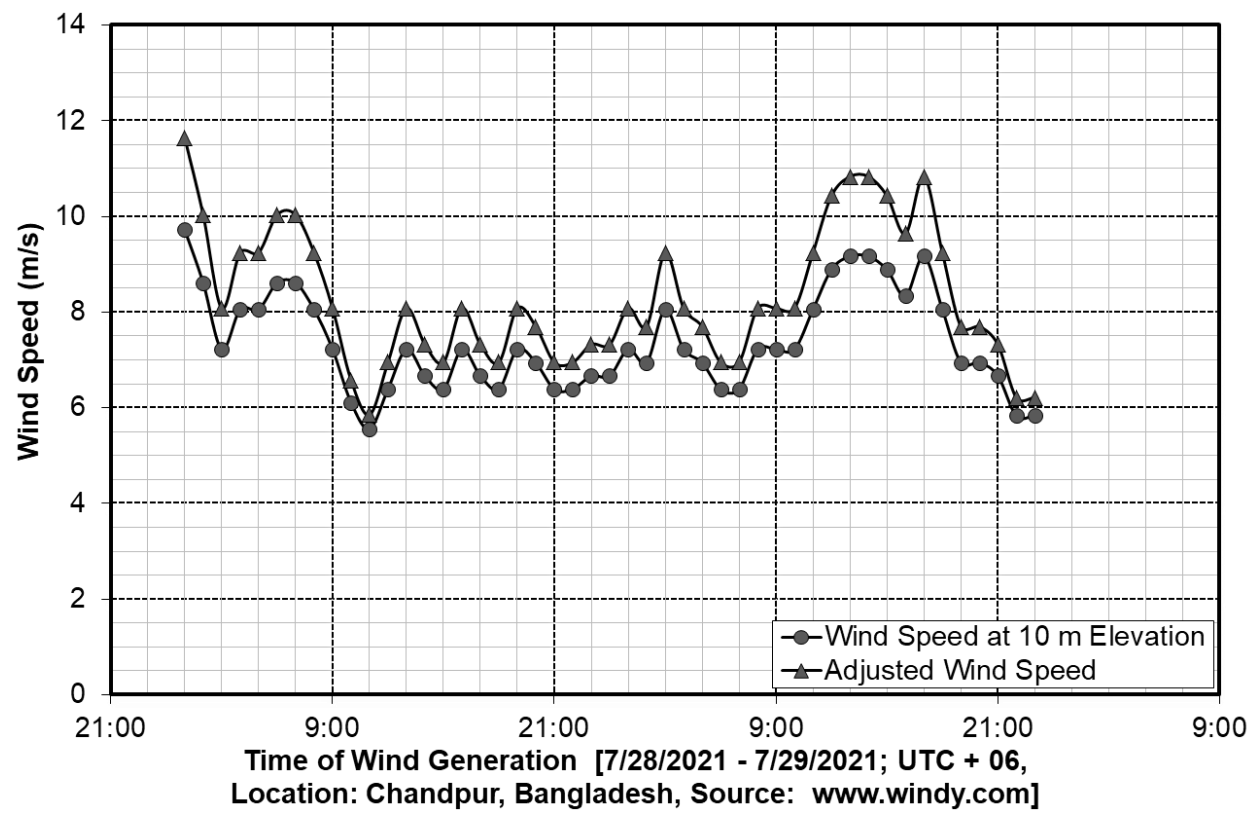

Fig. 3 Recorded wind speed and corresponding adjusted wind speed at time of generation

The wind speed data ( $U_{10}$ : wind velocity at $10 \mathrm{~m}$ elevation) from 0100 hours on $28^{\text {th }}$ July 2021 to 2300 hours on $29^{\text {th }}$ July 2021 have been recorded (local time: Bangladesh is in the time zone of UTC+06:00) (Figure 2). For having the wave generation from wind velocity, the wind stress (adjusted wind speed) has to be calculated first which is a function of drag 
On the Investigation of Wind Generated Waves in Bangladesh Rivers for the Assessment of Stability Requirements in Inland Vessel Design
Muhammad Rabiul Islam, Md. Mahmudul Hasan Akib, Fariha Tabassum, Khandaker Akhter Hossain

coefficient and wind speed as shown in equation 5 [7]. The wind speed at $10 \mathrm{~m}$ elevation and the corresponding adjusted wind speed are shown in Figure 3.

$$
U_{A}=0.71 U_{10}^{1.23}\left(U_{10} \text { in } \mathrm{m} / \mathrm{s}\right)
$$

\section{Fetch Length Calculation}

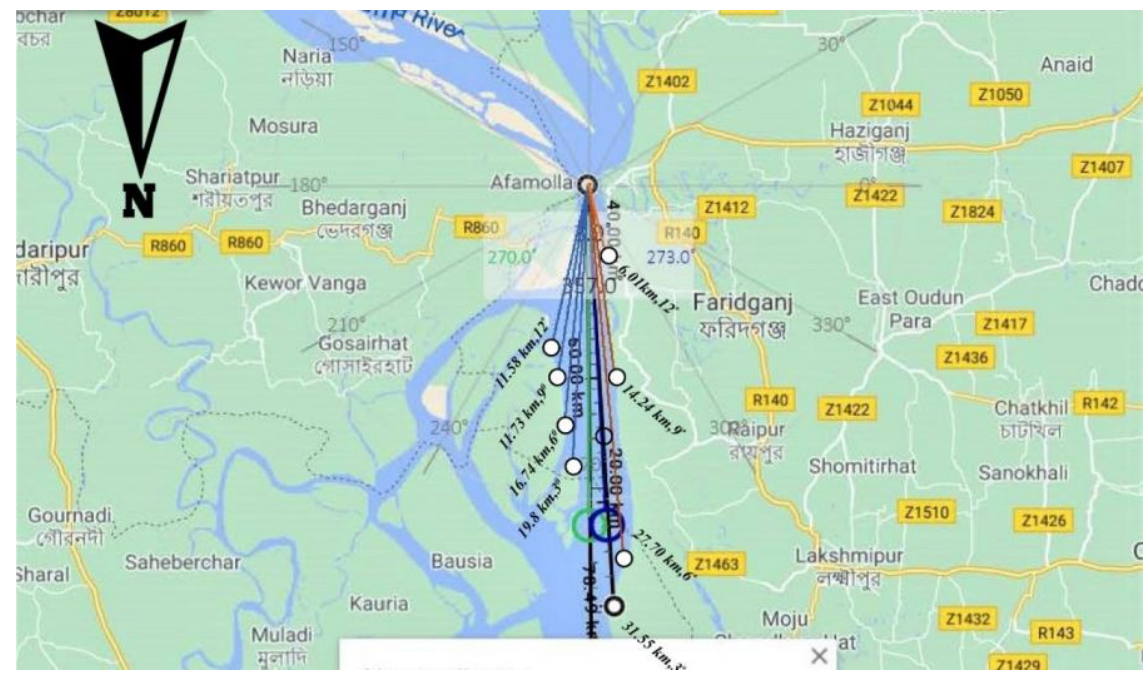

Fig. 4 Straight line fetches (Wind Dir $-0^{0} \mathrm{~N}$, Radial Distance $3^{0}$ ) (www.google.com/maps/)

Table 3 Different parameters of fetch length calculation as considered in this study

\begin{tabular}{|c|c|}
\hline Parameter & Value \\
\hline Wind Direction & 0 degree South \\
\hline Angle increment between radial measurements & 3 degree \\
\hline Direction of the first radial (measured clockwise from north) & 168 degree \\
\hline Number of radials & 9 \\
\hline Radial length for 168 degree & $11.58 \mathrm{~km}$ \\
\hline Radial length for 171 degree & $11.73 \mathrm{~km}$ \\
\hline Radial length for 174 degree & $16.74 \mathrm{~km}$ \\
\hline Radial length for 177 degree & $19.80 \mathrm{~km}$ \\
\hline Radial length for 180 degree & $46.94 \mathrm{~km}$ \\
\hline Radial length for 183 degree & $31.55 \mathrm{~km}$ \\
\hline Radial length for 186 degree & $27.70 \mathrm{~km}$ \\
\hline Radial length for 189 degree & $14.24 \mathrm{~km}$ \\
\hline Radial length for 192 degree & $6.01 \mathrm{~km}$ \\
\hline Simple Fetch (SPM, 1984) & $20.70 \mathrm{~km}$ \\
\hline Narrow Fetch (NARFET) & $28.38 \mathrm{~km}$ \\
\hline$\%$ of increase & 37.10 \\
\hline
\end{tabular}

Two different methods for calculating fetch length are considered in this study. Among them simple fetch is calculated following the guidance recommended by SPM, 1984. Here the 
Muhammad Rabiul Islam, Md. Mahmudul Hasan Akib, Fariha Tabassum, Khandaker Akhter Hossain
On the Investigation of Wind Generated Waves in Bangladesh Rivers for the Assessment of Stability Requirements in Inland Vessel Design

fetch is determined by arithmetically averaging straight-line fetches at increments of 3 degree over a 24-degree arc (12 degree either side of the mean wind direction) where the straight line fetches are measured from shoreline to the point of interest. This simple fetch method does not consider the fetch geometry and assumes that the wave direction coincides with the wind direction. The other method of fetch length (narrow fetch) calculation is done by a computer language program 'NARFET'.

Narrow fetch includes the option for consideration of narrow geometry of fetch by considering the wave generation in off-wind direction. Here fetch geometry is described by radial fetch lengths measured from the shoreline to the point of interest at even angle increments. The angle increment is usually small enough to resolve the shoreline, which is 3 degree in this study. Linear interpolation is used between the input values. Angles are measured clockwise from north and represent the direction of winds/waves are coming from. NARFET internally interpolates fetch lengths at 1-deg increments around the entire 360-deg arc or the input arc. Then the program averages fetch lengths over 15-deg arcs centered on each 1-degree increment. These fetch lengths are used to calculate wave conditions. The straight line fetches from shore line to the point of interest is shown in Figure 4. The fetch length output of 'NARFET' is $37.10 \%$ higher than the arithmetical average of straight line fetches (simple fetch) (Table 3).

\section{Assumed Standard Environmental Condition}

Following environmental conditions have been assumed in this study:

1. Wave conditions not fully developed;

2. Steady wind speed;

3. Steady wind direction;

4. Wave conditions not duration limited;

5. Water depths across the fetch are deep based on the peak frequency (depth is greater than half the wavelength);

6. No difference between air and sea temperature.

The shallow water waves that are generated in fetch-limited condition follow growth laws that are quite close to deep water wave growth for the same wind speeds, up to a point where an asymptotic depth-dependent wave height is attained. This suffices the disregard of bottom friction effects on wave growth in shallow water. Also, evidence indicates that wave spectra in shallow water do not appear to have a noticeable dependence on variations in bottom sediments. SPM, 1984 recommends to use deep water wave growth formulae for all depths subject to the constraint that the wave period should not exceed the following limiting value in case of shallow water waves.

$$
T_{p} \approx 9.78\left(\frac{d}{g}\right)^{\frac{1}{2}}
$$

In the above equation $\mathrm{T}_{\mathrm{p}}$ is the peak period, $\mathrm{d}$ the water depth and $\mathrm{g}$ is the gravitational acceleration.

\section{Significant wave height and peak period}

Significant wave height $\left(H_{S}\right)$ and peak period $\left(T_{P}\right)$ are the two very useful indexes to characterize the wave data on water surface. The average height of the one-third highest waves is termed as significant wave height. The wave parameter when considered based on 
On the Investigation of Wind Generated Waves in Bangladesh Rivers for the Assessment of Stability Requirements in Inland Vessel Design
Muhammad Rabiul Islam, Md. Mahmudul Hasan Akib, Fariha Tabassum, Khandaker Akhter Hossain

statistics of individual waves record is termed as statistical-based and when defined in terms of the standard deviation of sea surface elevations as represented by all data in a wave record is termed as energy-based. The latter one relates to the energy spectral density. On the other hand, peak period characterize the dominant periodicity of the waves. Alternately the period associated with the largest wave energy in the energy spectral density is termed as peak period which can only be obtained from spectral analysis. In this study waves of single peaked energy spectra are considered. The equations of energy based significant wave height $\left(H_{m 0}\right)$ and peak frequency $\left(f_{P}\right)$ derived from the JONSWAP experiment can be represented as shown in equation (7) and equation (8) respectively for the fetch limited wave condition [6].

$$
\begin{aligned}
& H_{m 0}=0.0016 g^{-0.5} F^{0.5} U_{10} \\
& f_{p}=3.5 g^{0.67} F^{-0.33} U_{10}^{-0.33}
\end{aligned}
$$

In the above equations $F$ is the simple fetch length as recommended by SPM, 1984. The values of energy based significant wave heights and the spectral peak periods corresponding to the wind velocity measured in this study have been calculated using equation (7) and (8). The values are plotted in Figure 5.

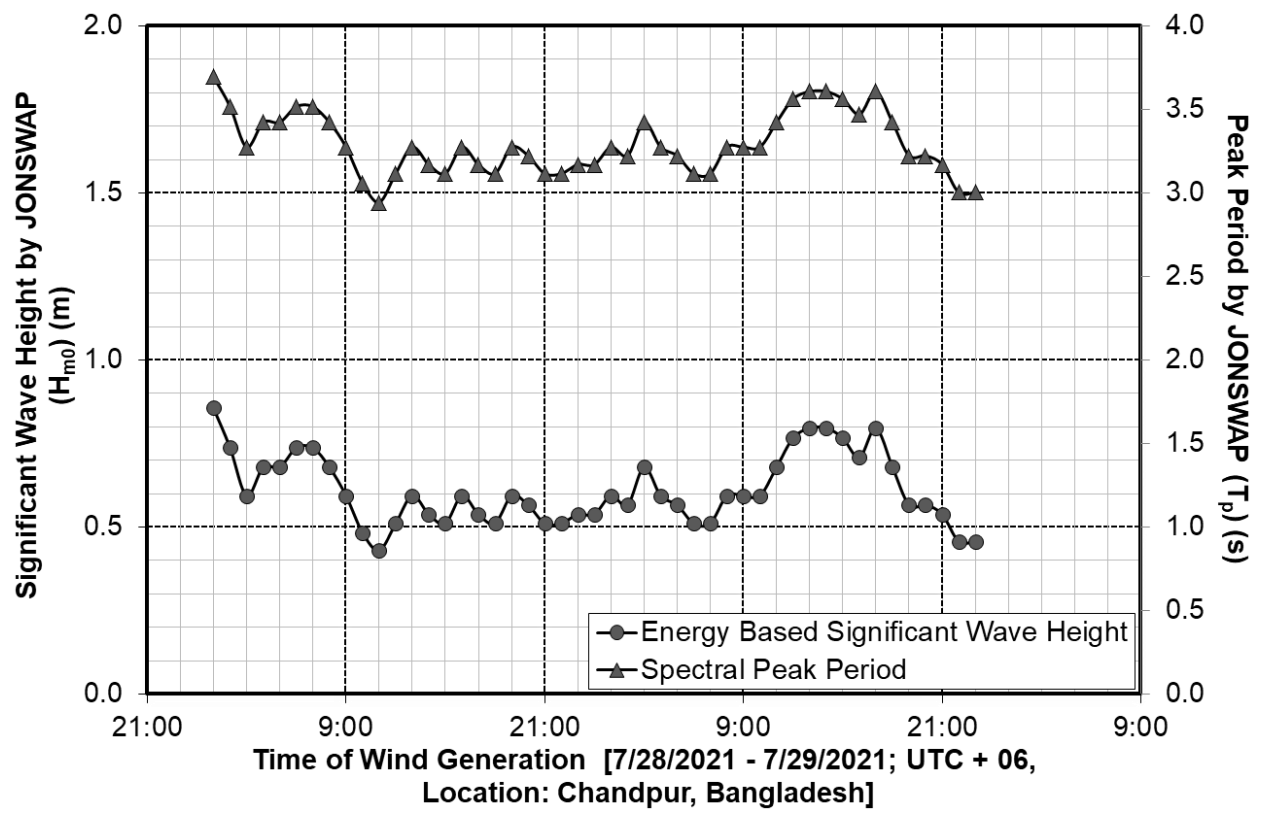

Fig. 5 Energy based significant wave height and spectral peak period for the recorded wind velocities by JONSWAP experiment

On the other hand the relations proposed by NARFET model are shown in equation (9) and in equation (10).

$$
\begin{aligned}
& H_{m 0}=0.0015 g^{-0.5} F^{0.5}\left(U_{10} \cos \phi\right) \\
& f_{p}=2.6 g^{0.72} F^{-0.28}\left(U_{10} \cos \phi\right)^{-0.44}
\end{aligned}
$$

In the above equations $F$ is the 15 degree averaged fetch length at an angle $\varphi$ with the wind direction considering the fetch geometry (narrow fetch) as calculated by the computer program 'NARFET'. The model produced by the 'NARFET' computer program is an improvement over the SPM, 1984 and Donelan [16] methods. The concept of wave development in off-wind directions, which was proposed by Donelan, is used in 'NARFET'. 
Muhammad Rabiul Islam, Md. Mahmudul Hasan Akib, Fariha Tabassum, Khandaker Akhter Hossain
On the Investigation of Wind Generated Waves in Bangladesh Rivers for the Assessment of Stability Requirements in Inland Vessel Design

The summarized approach of Donelan is balancing a reduced wind forcing $U \operatorname{Cos}(\varphi)$ with increased fetch distance in an off-wind direction. The 'NARFET' determines the direction of wave generation from the input wind direction by maximizing the wave period (reciprocal of equation (10)). The maximum period is achieved when

$$
(\cos \phi)^{0.44} F^{0.28}
$$

is maximized. When the fetch and angle that maximize equation (11) are determined, the fetch, angle, and wind speed are applied to equation (9) and equation (10) to calculate energy based significant wave height and peak period. The results of equation (9) and equation (10) are plotted in Figure 6.

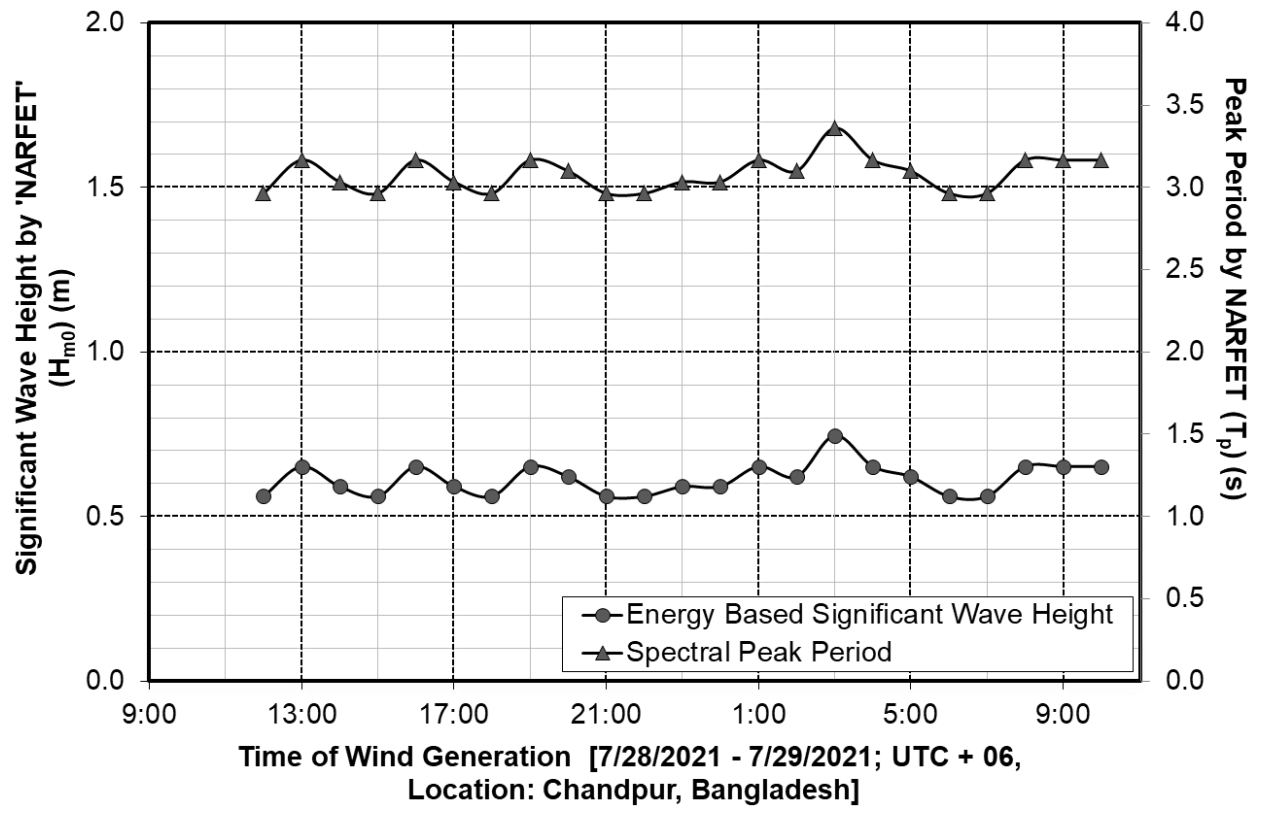

Fig. 6 Energy based significant wave height and spectral peak period for the recorded wind velocities by NARFET model

\section{Comparison between Simple Fetch and Narrow Fetch Methods}

In Figure 7, comparison between JONSWAP experimental relationships and improved model 'NARFET' is illustrated. From the plots it is seen that the simple fetch method tends to underestimate wave height and overestimate peak wave frequency. This result is exactly the same as the finding by Smith [6] which indicates the successful simulation of the 'NARFET' model. In the tests carried out by Smith, the simple fetch method underestimates wave height by as much as $40 \%$ for larger wave conditions which is $10 \%$ for the waves of current study. And for the peak period, the maximum overestimation by simple fetch method is $5 \%$ in current study.

The wave condition can better be represented by wave spectral analysis. It is already stated that the parametric spectrum model JONSWAP can reasonably be considered for inland navigational conditions. Comparison of mean energy spectral density by JONSWAP (equation (2)) between the two different methods of calculating peak frequency is illustrated in Figure 8 considering the average value of the peak enhancement factor $(\gamma=3.3)$. The average spectrum of a 22 hour portion (1200 hour on $28^{\text {th }}$ July 2021 to 1000 hour on $29^{\text {th }}$ July 2021) of the recorded wind velocity is plotted in Figure 8 considering the wind speed was relatively constant on that duration. The variation observed in the energy density of the two different methods is negligible. From the above discussions it can be decided that the 
On the Investigation of Wind Generated Waves in Bangladesh Rivers for the Assessment of Stability Requirements in Inland Vessel Design
Muhammad Rabiul Islam, Md. Mahmudul Hasan Akib, Fariha Tabassum, Khandaker Akhter Hossain

'NARFET' model can reasonably be chosen for investigating the wave parameters in Bangladesh inland navigational routes.

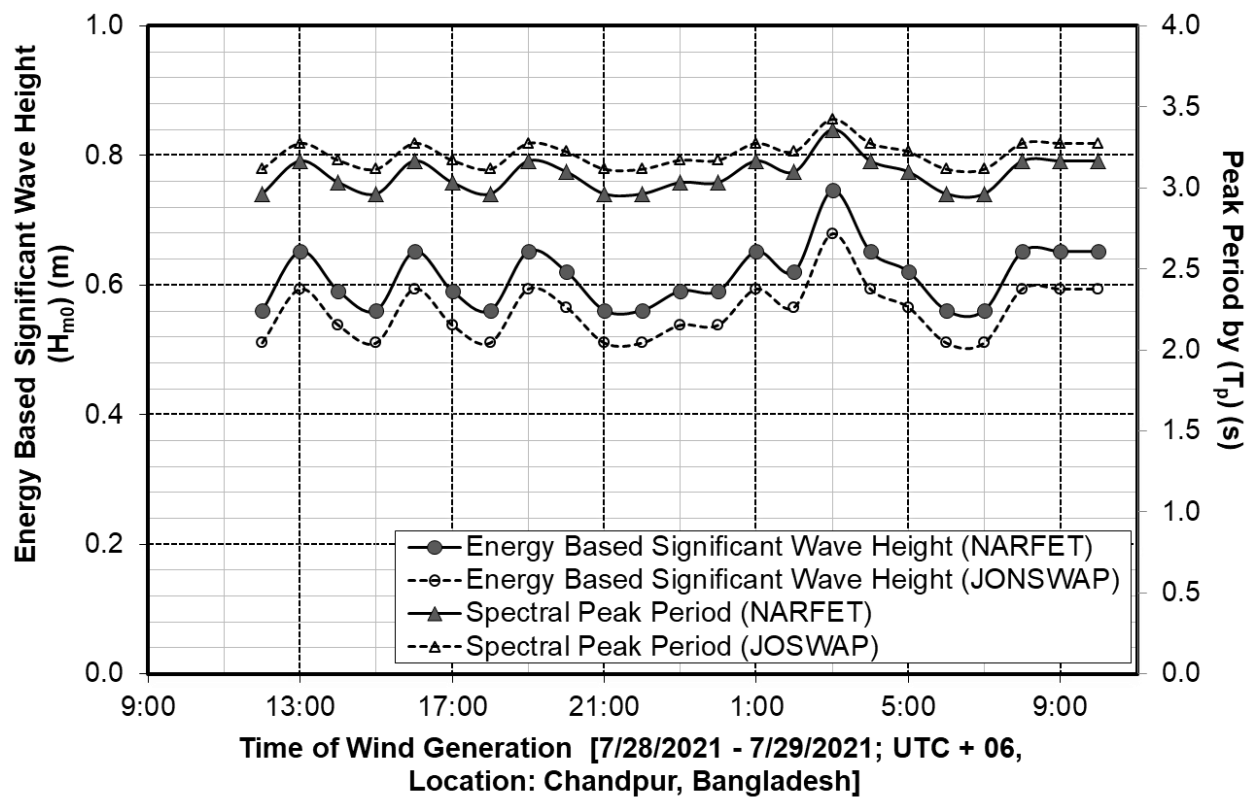

Fig. 7 Comparison between JONSWAP experimental relationships and improved model 'NARFET'

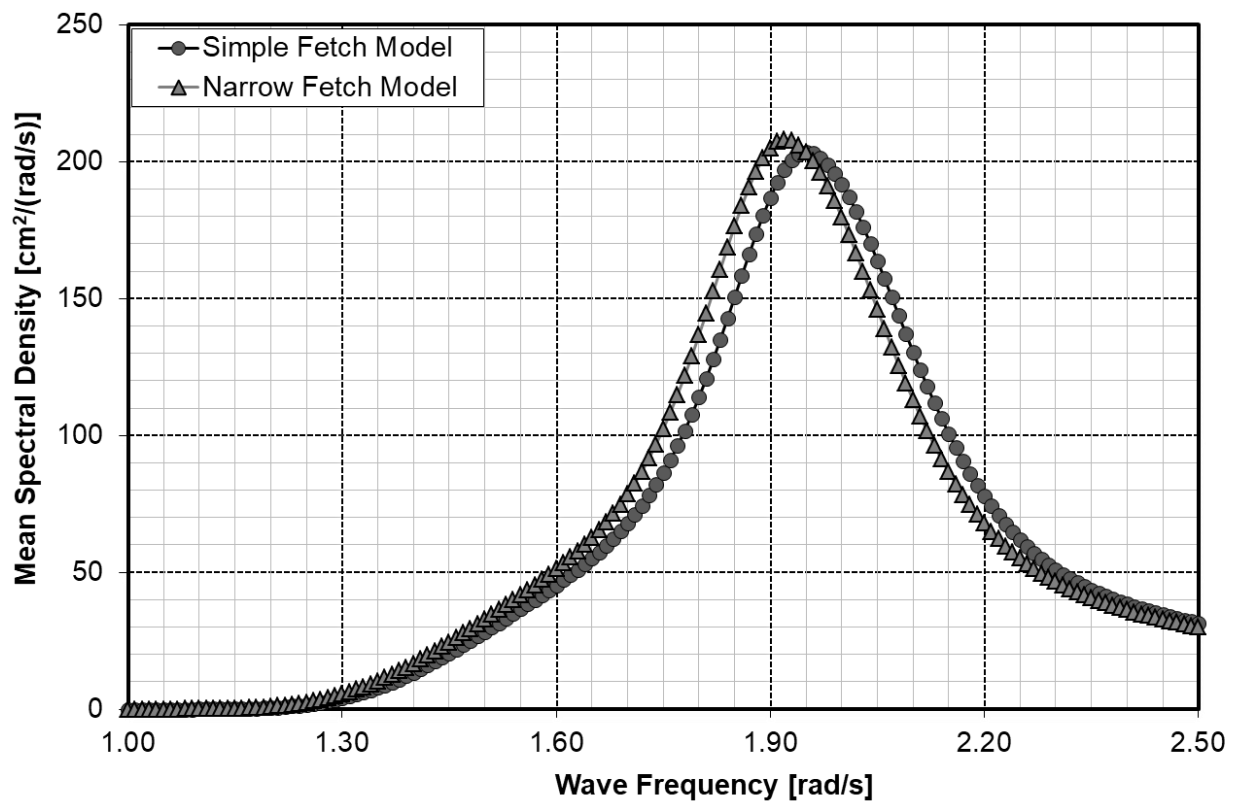

Fig. 8 Comparison of mean energy spectral density by JONSWAP between the two different models

\section{Wave Data corresponds to Design and Operation of Bangladesh Inland Vessel}

Wind speeds at different levels of signals in Bangladesh inland navigational route are shown in table 2. It is also stated before that the wind speed $20.5 \mathrm{~m} / \mathrm{s}$ corresponds to the specified wind pressure in the Bangladesh stability rules. Values of different wave parameters at those wind speeds are illustrated in Figure 9 where vertical solid line corresponds to wind speed $20.5 \mathrm{~m} / \mathrm{s}$ that is the design wind speed of Bangladesh domestic vessels. The figure demonstrates that fetch limited waves of significant height up to $2.40 \mathrm{~m}$ need to be considered 
Muhammad Rabiul Islam, Md. Mahmudul Hasan Akib, Fariha Tabassum, Khandaker Akhter Hossain
On the Investigation of Wind Generated Waves in Bangladesh Rivers for the Assessment of Stability Requirements in Inland Vessel Design

in designing the Bangladesh domestic vessels where the condition of fetch limitation will sustain up to nearly 3 (three) hours of wind blowing. The comparison between the spectral energy density is shown in Figure 10. Chatelier et. al. [9] have discussed existing applicable rules and regulations of different nations and also the Bureau Veritas rules regarding the inland navigation vessels especially the river-sea vessels. The emphasized common characteristic parameter is significant wave height. India, China and Russia have categorized their inland vessels depending on the wind and wave conditions on the basis of significant wave height, maximum $2 \mathrm{~m}$. Belgium and France also have their national regulation for riversea vessels and study of their coastal water also gives the value of significant wave height averaging $2 \mathrm{~m}$. The rules of Bureau Veritas that are applicable to inland vessels include specific notations based on the maximum significant wave height up to $2.0 \mathrm{~m}$.

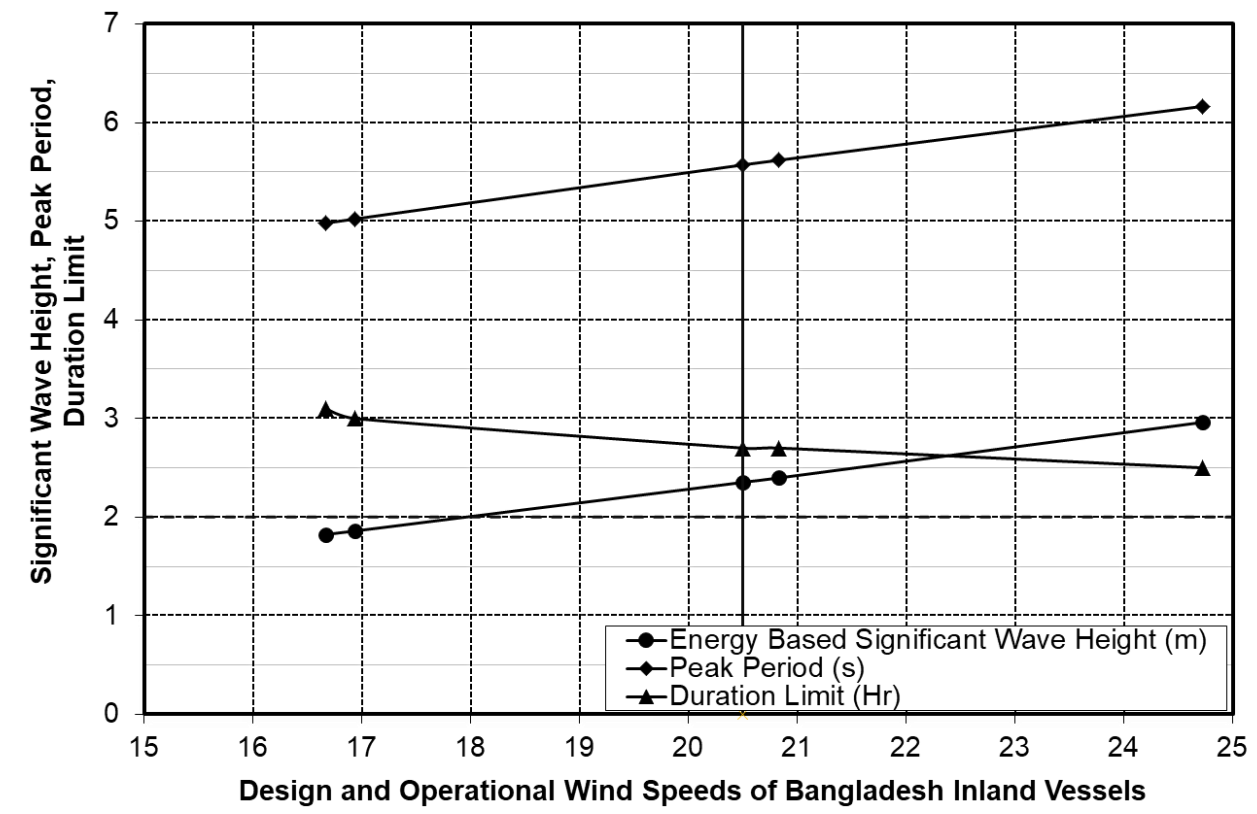

Fig. 9 Values of different wave parameters at design and operational wind speeds of Bangladesh Inland vessels

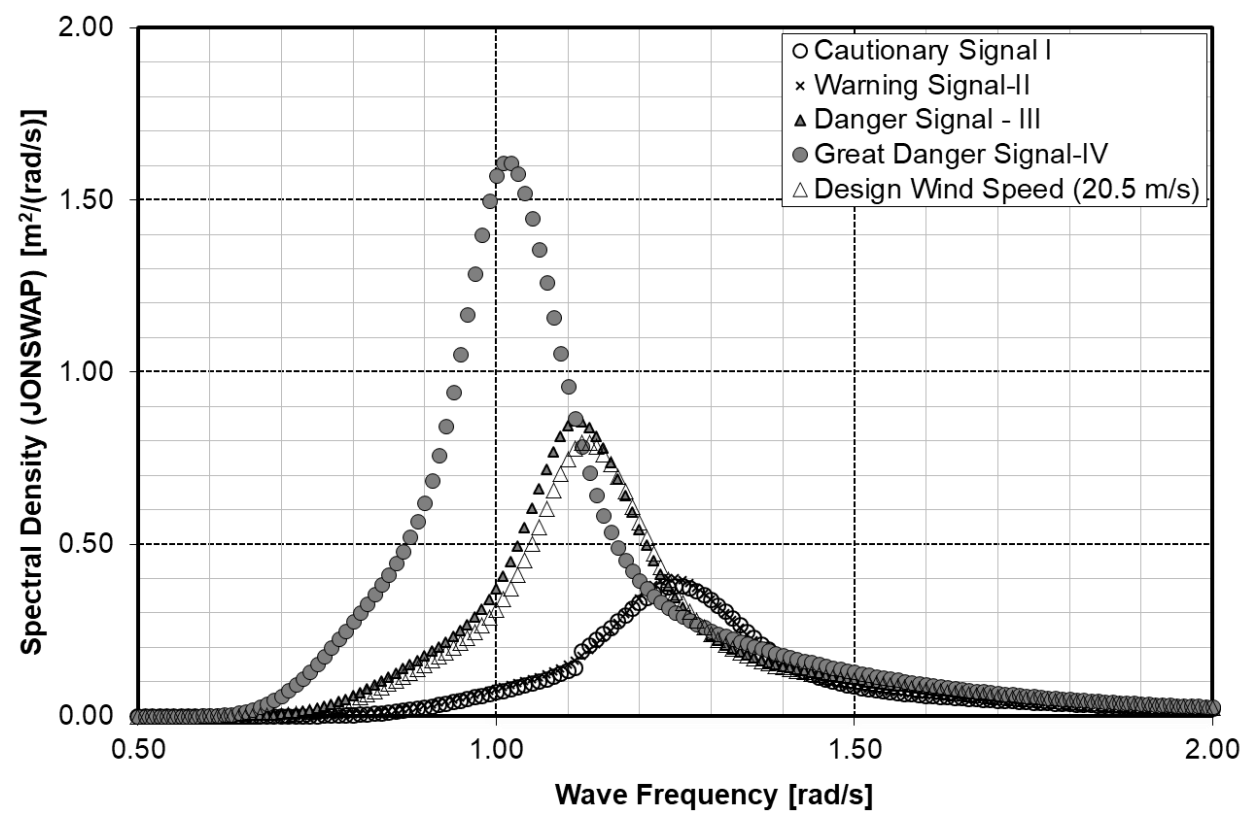

Fig. 10 Comparison of the spectral energy densities at different river conditions 
On the Investigation of Wind Generated Waves in Bangladesh Rivers for the Assessment of Stability Requirements in Inland Vessel Design
Muhammad Rabiul Islam, Md. Mahmudul Hasan Akib, Fariha Tabassum, Khandaker Akhter Hossain

It is very much clear from the results of this study that wind and wave conditions of Bangladesh inland river routes have a significant effect on the vessel's stability requirement. Bangladesh inland vessels should also be characterized by a significant wave height of $2 \mathrm{~m}$ as a first approximation. The stability and seaworthiness of these vessels must be in accordance with the rules as specified by the class for such category of vessels and equivalent of the socalled river sea vessels.

\section{Consideration for Second Generation Intact Stability Criteria}

For investigating the performance based dynamic stability failure modes as specified by the SGISC, knowledge regarding the wave load is must. The interest for studying the dynamic failure modes in inland navigation is rarely shown by the researchers. It is understandable that the international agencies or organizations cannot give that much emphasize on the inland navigational routes or vessels. That may be due to the fact that the infinite depth of water, extreme weather impact and large but identical wave characteristics in seaways can bring the world in a single platform from the viewpoint of minimum stability requirement of a ship. On the other hand the heterogeneity of parameters [including water depth, socio economic condition, local extreme weather, geometry of water bodies etc.] governing the stability requirement for safety of ship in inland waterways demands individual efforts of countries. This study clearly indicates the necessity of investigating the dynamic failure modes in Bangladesh inland river routes at least for the medium size vessels which are mainly accident prone. It is mentioned before that the Interim guidelines on the SGIC, the short-term characterization of standard environmental conditions has been assumed by Bretschneider energy spectrum. Figure 11 illustrates the comparison of energy spectral density between the JONSWAP (equation 2) and Bretschneider (equation 4) for the same river condition corresponding to Bangladesh river danger signal III (wind speed $20.83 \mathrm{~m} / \mathrm{s}$ ).

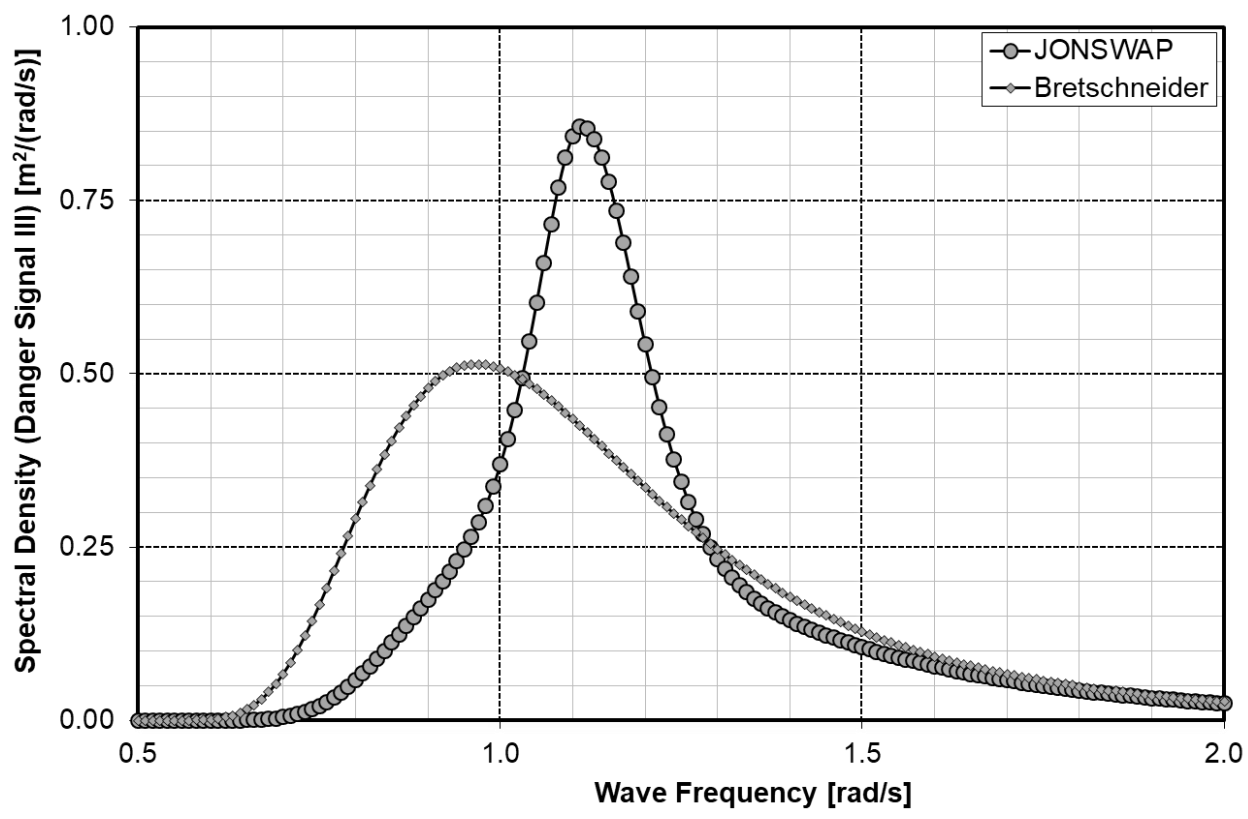

Fig. 11 Comparison of energy density between the JONSWAP spectrum and Bretschneider spectrum

In SGISC, the long-term characterization of standard environmental condition is given in terms of a table containing the probabilities of each range of sea states encountered in the considered operational area or operational route called wave scatter table. The table is formed on the basis of North Atlantic data focusing on extreme wave loads. A limited wave scatter table which is obtained from the full wave scatter table by removing all sea state ranges with 
Muhammad Rabiul Islam, Md. Mahmudul Hasan Akib, Fariha Tabassum, Khandaker Akhter Hossain
On the Investigation of Wind Generated Waves in Bangladesh Rivers for the Assessment of Stability Requirements in Inland Vessel Design

the significant wave height above a certain limit can successfully characterize river routes. SGISC emphasizes on combined consideration of design and operational aspects for achieving a sufficient safety level and has given the freedom to local administration of accepting alternative environmental conditions for ships subject to operational measures.

It is suggested that the alternative environmental conditions should specify the shortterm characteristics of wind and sea state, together with the probability of occurrence of each short-term environmental condition. The river state recommended for Bangladesh inland vessel's design in current study and the calculated corresponding characteristic wave parameters considering a crucial location (Chandpur triangle) of Bangladesh inland river route are shown in Table 4. A wave scatter table consisting of number of occurrences of waves within each range of significant wave height up to $2.4 \mathrm{~m}$ may form considering the other parameters that are shown in Table 4.

Table 4 Recommended short term river condition in designing Bangladesh inland vessel

\begin{tabular}{|c|c|}
\hline Parameter & Value \\
\hline River signal & Danger Signal No. III \\
\hline Wind speed & $75 \mathrm{~km} / \mathrm{h}(20.83 \mathrm{~m} / \mathrm{s})$ \\
\hline Fetch & Narrow \\
\hline Significant wave height & $2.40 \mathrm{~m}$ \\
\hline Significant wave period & $5.32 \mathrm{~s}$ \\
\hline Peak period & $5.62 \mathrm{~s}$ \\
\hline Average zero crossing period & $4.40 \mathrm{~s}$ \\
\hline
\end{tabular}

\section{Conclusion}

The capsize sensitivity of medium size passenger vessels of Bangladesh in stormy weather demands the assessment of failure modes due to loss of dynamic stability. Understanding the dynamics of vessels requires knowledge of environmental load which is wind and wind generated waves in inland navigational routes. The wind wave parameters of a crucial location in Bangladesh inland waterways have been estimated in this study considering the wind velocities from a reliable secondary source. Fetch limited wave energy spectrum along with the consideration of river route geometry by considering fetch geometry has been used to assess the Bangladesh river state. Considering the operational limits related to maximum significant wave height, it is seen that the minimum stability requirement of Bangladesh inland vessels should at least be equivalent to river-sea vessels as defined by different other nationals and the classification societies. Alternative environmental conditions need to be developed for Bangladesh inland vessels design consideration for both the IMO IS Code, 2008 and the SGISC.

\section{Recommendation}

The wave forecasting models usually cannot supplant wave measurements. These are recommended in developing wave conditions for design and planning situations having safety implications and should be properly verified with local wave data (if feasible). Direct measurement of real wind data with validation of the applicable wave forecasting model followed by the proposed method in this study can be considered in developing an alternative environmental condition for deciding the minimum stability requirements of inland vessel design, especially for Bangladesh. 
On the Investigation of Wind Generated Waves in Bangladesh Rivers for the Assessment of Stability Requirements in Inland Vessel Design
Muhammad Rabiul Islam, Md. Mahmudul Hasan Akib, Fariha Tabassum, Khandaker Akhter Hossain

\section{REFERENCES}

[1] IMO, Maritime Safety Committee (2008): Adoption of the International Code on Intact Stability, 2008 (2008 IS Code), Resolution MSC.267 (85), London

[2] IMO, Sub-committee on ship design and construction (2020): Finalization of Second Generation Intact Stability Criteria, Report of the Drafting Group on Intact Stability, 7th Session, Agenda Item 5(SDC 7/6)

[3] Iqbal, K.S., Bulian, G., Hasegawa, K., Karim, M.M., Awal, Z.I. (2008): A rational analysis of intact stability hazards involving small inland passenger ferries in Bangladesh, Journal of marine science and technology, 13(3), pp.270-281 https://doi.org/10.1007/s00773-008-0016-3

[4] Islam, M.R., Rahaman, M.D., Degiuli, N. (2015): Investigation of the causes of maritime accidents in the inland waterways of Bangladesh, Brodogradnja, 66(1), pp.12-22

[5] U.S. Army Corps of Engineers (2002): Coastal Engineering Manual, Engineer Manual 1110-2-1100 (6 volumes), Washington, D.C.

[6] Smith, J.M. (1991): Wind-wave generation on restricted fetches, Coastal Engineering Research Center, US

[7] U.S. Army Corps of Engineers (1984): Shore Protection Manual (1), Coastal Engineering Research Center, US

[8] Ozeren, Y.A.V.U.Z., Wren, D.G. (2009): Predicting wind-driven waves in small reservoirs, Transactions of the ASABE, 52(4), pp.1213-1221 https://doi.org/10.13031/2013.27793

[9] Chatelier, J.M., Nzengu, W.N., Cocito, R. (2017): Stability and seakeeping of river-sea vesselsclassification rules, Proceedings of ISSW, pp. 149-158

[10] Sverdrup, H.U., Munk, W.H. (1947) Wind, sea and swell: Theory of relations for forecasting, Hydrographic office publication no.601 https://doi.org/10.5962/bhl.title.38751

[11] Yamagata, M. (1959) Standard of Stability Adopted in Japan, Transaction of the Institution of Naval Architects, 101, pp. 417-443

[12] IMO, Sub-Committee on Stability and Load Lines and on Fishing Vessels Safety (2008): Report of the intersessional Correspondence Group on Intact Stability, Document SLF 51/4/1, submitted by Germany

[13] Chakrabarti, S.K. (1987): Hydrodynamics of offshore structures, WIT press

[14] Windy: Wind Map \& Weather Forecast

URL: https://www.windy.com/

[15] Vinayak, P.P., Prabu, C.S.K., Vishwanath, N. i Prakash, S.O. (2021): Numerical simulation of ship navigation in rough seas based on ECMWF data, Brodogradnja, 72 (1), pp.19-58 https://doi.org/10.21278/brod72102

[16] Donelan, H. A. (1980): Similarity Theory Applied to the Forecasting of Wave Heights, Periods, and Directions, Proceedings of the Canadian Coastal Conference. National Research Council, pp 47-61

Submitted: $\quad$ 18.08.2021. Corresponding Author: Muhammad Rabiul Islam, PhD rabiul@name.mist.ac.bd

Accepted: $\quad$ 10.09.2021. Faculty, Naval Architecture and Marine Engineering Department Md. Mahmudul Hasan Akib, akib.name06@gmail.com Graduating Student, Naval Architecture and Marine Engineering Department Fariha Tabassum, farihtabassum98@gmail.com Graduating Student, Naval Architecture and Marine Engineering Department Khandakar Akhter Hossain, PhD, akhter756@name.mist.ac.bd Faculty and Head, Naval Architecture and Marine Engineering Department Military Institute of Science and Technology (MIST)

Dhaka, Bangladesh 